\title{
Patient-centred tuberculosis treatment delivery under programmatic conditions in Tanzania: a cohort study Saidi Egwaga ${ }^{1}$, Abdallah Mkopi ${ }^{2}$, Nyagosya Range ${ }^{3}$, Vera Haag-Arbenz ${ }^{4}$, Amuri Baraka², Penny Grewal ${ }^{4}$, Frank Cobelens ${ }^{5,6}$, Hassan Mshinda ${ }^{2}$, Fred Lwilla ${ }^{1}$ and Frank van Leth $* 5,6$
}

\begin{abstract}
Address: ${ }^{1}$ National Tuberculosis and Leprosy Programme, Ministry of Health and Social Welfare, Dar es Salaam, Tanzania, ${ }^{2}$ Ifakara Health Institute, Ifakara, Tanzania, ${ }^{3}$ National Institute for Medical Research, Dar es Salaam, Tanzania, ${ }^{4}$ Novartis Foundation for Sustainable Development, Basle, Switzerland, ${ }^{5} \mathrm{KNCV}$ Tuberculosis Foundation, The Hague, The Netherlands and ${ }^{6}$ Center for Infection and Immunity, Academic Medical Center, University of Amsterdam, Amsterdam, The Netherlands

Email: Saidi Egwaga - tantci@intafrica.com; Abdallah Mkopi - amkopi@ihrdc.or.tz; Nyagosya Range - hrange08@gmail.com; Vera HaagArbenz - vera.haag_arbenz@ novartis.com; Amuri Baraka - amuribaraka@ihrdc.or.tz; Penny Grewal - grewalp@mmv.org;

Frank Cobelens - CobelensF@kncvtbc.nl; Hassan Mshinda - hassanmshinda@ihrdc.or.tz; Fred Lwilla - flwilla@yahoo.com; Frank van Leth* - vanlethf@kncvtbc.nl

* Corresponding author
\end{abstract}

Published: 21 December 2009

BMC Medicine 2009, 7:80 doi:10.1 186/1741-7015-7-80

This article is available from: http://www.biomedcentral.com/ I74I-70I5/7/80

(C) 2009 Egwaga et al; licensee BioMed Central Ltd.

This is an Open Access article distributed under the terms of the Creative Commons Attribution License (http://creativecommons.org/licenses/by/2.0), which permits unrestricted use, distribution, and reproduction in any medium, provided the original work is properly cited.
Received: 19 November 2009

Accepted: 21 December 2009

\begin{abstract}
Background: Directly observed therapy (DOT) remains the cornerstone of the global tuberculosis (TB) control strategy. Tanzania, one of the 22 high-burden countries regarding TB, changed the first-line treatment regimen to contain rifampicin-containing fixed-dose combination for the full 6 months of treatment. As daily health facility-based DOT for this long period is not feasible for the patient, nor for the health system, Tanzania introduced patient centred treatment (PCT). PCT allows patients to choose for daily DOT at a health facility or at their home by a supporter of choice. The introduction of fixed dose combinations in the intensive and continuation phase made PCT feasible by eliminating the risk of selective drug taking by patients and reducing the number of tablets to be taken. The approach was tested in three districts with the objective to assess the effect of this strategy on TB treatment outcomes
\end{abstract}

Methods: Cohort analysis comparing patients treated under the PCT strategy (registered April-September 2006) with patients treated under health-facility-based DOT (registered April-September 2005). The primary outcome was the cure rate. Differences were assessed by calculating the risk ratios. Associations between characteristics of the supporters and treatment outcomes in the group of patients opting for home-based DOT were assessed through logistic regression.

Results: In the PCT cohort there were 1208 patients and 1417 were included in the historic cohort. There was no significant difference in cure rates between the cohorts (risk ratio [RR]: I.06; 95\% confidence interval [CI]: 0.96-I.I6). In the PCT cohort, significantly more patients had successful treatment (cure or treatment completed; $\mathrm{RR}$ : I.I0; $95 \% \mathrm{Cl}$ : I.0I-I.I5). There were no characteristics of supporters that were associated with treatment outcome.

Conclusion: The PCT approach showed similar cure rates and better treatment success rates compared to daily healthfacility DOT. The results indicate that there are no specific prerequisites for the supporter chosen by the patient. The programmatic setting of the study lends strong support for scaling-up of TB treatment observation outside the health facility. 


\section{Background}

Tanzania is one of the 22 high-burden countries with respect to the number of incident tuberculosis (TB) cases[1]. Up to the mid-eighties of the previous century, the annual number of cases identified and treated in the country was manageable, but due to the developing epidemic with the human immunodeficiency virus (HIV), the caseload in Tanzania increased rapidly. Within 5 years, the number of TB cases notified tripled from 5000 to 15,000 between 1980 and 1985. In 2006, Tanzania reported over $62,000 \mathrm{~TB}$ cases (157 cases per 100,000 population), while the World Health Organization (WHO) estimated the incidence to be 312 per 100,000 population [1]. Thirty-five percent of smear-positive TB patients were HIV-positive in a recent study [2].

The currently recommended strategy for TB control is the directly observed treatment, short course (DOTS) $[3,4]$. This strategy includes, amongst others, the daily observation of patients' drug intake at the treating facility during the initial 2 months of therapy. The reason behind this observation is that it will ensure adequate treatment adherence during the period, in which the treatment regimen contains rifampicin which will prevent the selection of Mycobacterium tuberculosis strains resistant to this powerful drug [5-8].

The National Tuberculosis and Leprosy Programme (NTLP) of Tanzania changed the standard first-line treatment regimen in 2006 from an 8-month regimen to a 6month regimen. In the latter, both the intensive phase treatment and the continuation phase treatment contains rifampicin, while in the former rifampicin is only given for 2 months of the intensive phase. The rifampicin-containing regimen throughout the course of treatment has a higher efficacy in situations with a high prevalence of HIV-infection in the general population [9-11]. The WHO recommends the observation of treatment throughout the 6 months of this regimen at least three times a week [3]. This is clearly not feasible at the health facility level for either the patient or the health staff. Initial studies in urban and rural areas of Tanzania showed that decentralized treatment observation by guardians or former TB patients was feasible without a detrimental effect on treatment outcome $[12,13]$. The main drawback encountered in a possible scale-up of this approach was the need for incentives expressed by the supporters which made the strategy non-sustainable. The experiences from these studies formed the basis of a new approach to the implementation of DOTS in Tanzania in which the observation of treatment intake could be transferred from the health facility to the patient's home and the observer could be changed from a health-care worker to a supporter of choice.
These changes maintain the core principle of daily observation of treatment (DOT) for the full duration of treatment, prevent health facilities from being overburdened and enable the patients to choose a supervision model within the constraints of their daily life. This should have a positive effect on treatment adherence and, subsequently, the efficacy of treatment. The new strategy was labelled 'patient centred treatment' (PCT).

From previous studies it is known that DOT outside the health facility, by either health personnel or laymen, is feasible and can result in treatment outcomes that are similar to those under conventional DOT at the health facility [14-18]. In Nepal, both DOT at the health facility and DOT by family members obtained success rates that met the international target of $85 \%$ [17]. However, most of these studies have been designed as randomized clinical trials and/or put restrictions on the type of supporter who can observe treatment intake. Clinical trials have the inherent limitation of coming with increased supervision and monitoring which can have a positive effect of its own on treatment delivery and adherence. Restrictions on supporters complicate the interpretation and replication of results within the context of national control programmes with a wider choice of potential treatment supporters. Thus, there is an urgent need to test the concept of delegating treatment observation outside the health facility under routine programmatic conditions. This need also holds true for fields outside the context of TB where strategies other than health facility-based treatment delivery are being examined, such as home-base care programmes for the delivery of antiretroviral therapy to HIV-infected patients.

The concept of PCT was tested and well perceived by patients and health care professionals when assessed in a qualitative survey. Furthermore, the proposed strategy was seen to be a positive contribution to adherence to therapy [2]. The strategy was formally tested in three districts under programmatic conditions with no restriction on the choice of treatment supporter. The objective of this study was to assess whether favourable treatment outcomes (cure and success) in new patients (that is, those without previous $\mathrm{TB}$ treatment) treated under the new strategy were no lower than those in new patients who were treated under the conventional strategy. If so, this would lend strong support to the practice of observing treatment intake outside the health facility by laymen, under programmatic conditions.

\section{Methods \\ Design}

Initially, the study was designed as a cohort study comparing treatment outcomes for patients who chose homebased treatment with those who chose health facility- 
based treatment. This design was based on the results from a community assessment in which patients, if given a choice, were asked what would be their preference for the place of treatment. In this assessment, just over $50 \%$ would have opted for home-based treatment [2]. During the implementation of the current study it became clear that a much higher proportion of patients opted for home-based treatment, making a formal comparison of treatment outcomes with patients opting for health facility-based treatment statistically more difficult.

The study design was changed into a comparison of treatment outcomes for patients in the PCT cohort with those in a control cohort of all registered new TB patients in the same facilities during the same period of time a year earlier. This also changed the intervention being tested because three variables in the PCT cohort were different from the control cohort; the possibility of having homebased treatment observation, treatment with fixed dose combinations (FDCs) and a regimen with rifampicin for the continuation phase resulting in 6 months duration instead of 8 months. Possible differences between the cohorts should, therefore, be attributed to the overall intervention of PCT, rather than only the home-based setting of treatment observation.

\section{Study population and setting}

The study was carried out in the Arusha Municipality, the Kahama district (Shinyanga province) and the Mufindi district (Iringa province) of Tanzania. The decision to choose these sites was based on the number of notified smear-positive TB-patients and their representativeness for urban and rural settings in the country. Within each area, all TB treatment facilities implemented PCT for all new patients who were registered in the second and third quarter of 2006. The only inclusion criterion used was that the patient was defined as 'new', indicating that he/ she had never received any TB-treatment that lasted longer than 1 month. The control cohort comprised all new patients in the same health facilities registered in the second and third quarter of 2005 who were treated under the conventional DOTS strategy. Smear-positive TB was recorded when two out of three sputum samples (spot, morning, spot) were Ziehl-Neelsen positive. When clinical symptoms were suggestive of TB, but sputum samples were negative, a suggestive chest X-ray could lead to the diagnosis of smear-negative TB.

\section{Intervention}

The intervention tested was PCT, which consisted of three components. First, each patient was given the choice to receive treatment at home observed by a supporter of his/ her choice or to receive daily treatment at the health facility observed by health staff. There were no restrictions on the choice of supporter. Second, treatment for all patients was delivered as a FDC rather than the conventional separate tables for each drug. Third, the treatment contained isoniazid, rifampicin, ethambutol and pyrazinamide in the initial phase of 2 months and isoniazid and rifampicin in the continuation phase of 4 months. At any time, patients were allowed to change from home-based treatment to health facility-based treatment or vice versa. There were no interventions related to default tracing and monitoring in the PCT strategy, other than the routine guidelines from the NTLP, making the control cohort and the PCT cohort comparable in this respect.

\section{Follow-up and data collection}

If the patient opted for home-based treatment, he/she was asked to return with the supporter of his choice. The supporter was given instruction by the health care provider on the importance of daily supervision of drug intake, the signs and symptoms of side-effects, and what to do if they occur, and the frequency of the collection of new drugs. Supporters needed to escort the patient to the health facility on a weekly basis in the first 2 months of treatment to collect new drugs, report on the well-being of the patient and to discuss any problem encountered in the support of the patient. The patient was requested to join the supporter at every visit unless too ill to do so. In the remaining 4 months, the visits to the health facility took place twice a month.

Data on the demographics of patients and supporters, drug intake, side effects and laboratory results in the PCT cohort were prospectively recorded in specifically designed registers and cards. Patient and laboratory data in the control cohort were retrospectively retrieved from the TB registers and the patient's treatment cards in the participating health facilities. These routine registers were checked for accuracy (and updated if needed) by the same independent team that collected data in the PCT cohort in order to minimize ascertainment bias. Data collection for both cohorts took place in three rounds from September 2006 to July 2007. Follow-up ended when the last included patient reached the time of treatment completion (April 2007). This meant that the last data collection took place after the treatment outcome of all patients had been recorded.

\section{Outcome measures and explanatory variables}

The primary study outcome was the proportion of new smear-positive patients cured at the end of treatment $(6$ months in the PCT cohort and 8 months in the control cohort). Secondary outcomes were the proportion of patients with treatment success (cured or treatment completed) at 6 months or 8 months, and the proportion of smear-positive patients with smear conversion at 2 months. In addition, we assessed whether the characteristics of the treatment supporters were associated with treat- 
ment outcomes in the group of patients with home-based treatment observation. Cure was defined as a smear-positive patient having a negative sputum smear at the end of treatment and on at least one previous occasion. Treatment completion was defined as a patient having completed treatment but not having a final smear examination. The characteristics of the supporters were categorized based on distribution with the purpose of getting groups of similar size. Age in years was defined as $<25,25-34,35-44$ and $\geq 45$. The relationship with the patient was categorized as 'family member' or 'non family member'. Education was categorized as 'none', 'primary' and 'above primary'. The initially collected variable 'household of supporter' was not used due to the strong collinearity with the variable 'relationship' in the analyses.

\section{Quality control}

All sputum smears during diagnosis and follow-up were confirmed by an independent laboratory technician. Discrepancies between the initial test and the re-reading were resolved by a third reader whose results were final.

\section{Statistical analyses}

Baseline characteristics of the two cohorts were compared using the $\chi^{2}$ test for binary variables and Student's $t$-test for continuous variables. Outcome measures in the cohorts were compared by calculating the risk ratio (RR) stratified by district. A combined analysis of all three districts was only performed when there was no effect modification by district as assessed by the Cochran-Mantel-Haenszel test.

Supporter characteristics associated with cure and treatment success in the group of patients with home-based treatment observation were assessed by univariable and multivariable logistic regression. All multivariable analyses included, apart from 'place of treatment' the variable 'district' to incorporate the stratified design in the analyses. Other variables were only included if they were significantly associated with the outcome in univariable analyses with a $P$-value of 0.1 or less. Effect modification by districts in these models was assessed by testing for interactions (multiplicative). Effect modification was assumed statistically significant at the $10 \%$ level.

\section{Missing data and loss to follow-up}

Due to an unforeseen logistical problem, the cards designed for recording information on the supporters were not available for the first 205 patients. Unfortunately, not all information could be retrieved at a later stage. This did not introduce a bias as the study started in all the three districts at the same time. Furthermore, the patients' freedom of choice for the type of treatment delivery strategy remained. The missing cards only reduced the power of the study for the analyses assessing the effects of supporter characteristics on treatment outcomes in the PCT cohort.
The missing data were categorized as a separate level in the respective variables in order to include all treatment outcomes in the analyses. Therefore, statistical significant associations were assessed on level the Wald test in the logistic regression models rather than the likelihood ratio test.

\section{Sample size}

Based on the total number of new smear-positive patients who opted for home-based treatment and the corresponding number of patients a year earlier, the power of the study for the primary outcome was an $80 \%$ ability to detect a statistically significant difference $(\alpha=5 \%)$ of $8.5 \%$ when cure in the control cohort was estimated at $70 \%$.

\section{Ethics statement}

The study was approved by the Institutional Review Board of the Ifakara Health Institute in Tanzania. Informed consent was not obtained from the participants as the intervention was based on a change in national treatment guidelines that was applied to all.

\section{Role of the funding source}

The funder of the study was involved in the study design and data interpretation. Data collection and data analysis were performed independently of the funder. The corresponding author had access to all data in the study. The final decision to submit for publication was made by the NTLP of Tanzania.

\section{Results}

The study included 1208 new patients in the PCT cohort and 1417 in the control cohort. Of these, 548 (38.7\%) and $484(40.1 \%)$ were smear-positive (Figure 1). The proportion of females and the age of the patients were comparable in both cohorts (Table 1). The relative contribution of the districts for both cohorts differed: there were slightly more patients from the urban setting in the PCT cohort (40\%) than in the control cohort $(30 \%)$. Smear-negative patients were slightly under-represented in the PCT cohort compared to the control cohort. None of the analyses showed an interaction by district. Therefore, the outcomes were pooled and analysed for the total study population.

\section{Comparing treatment outcomes between cohorts}

Treatment outcomes comparing both cohorts are summarized in Table 2.

\section{Smear-positive patients}

None of the smear-positive patients in the PCT cohort who had a smear examination at the end of treatment had a positive smear result at the end of treatment, compared to two in the control cohort. This implies that differences in cure rate were driven by the presence of a smear result 


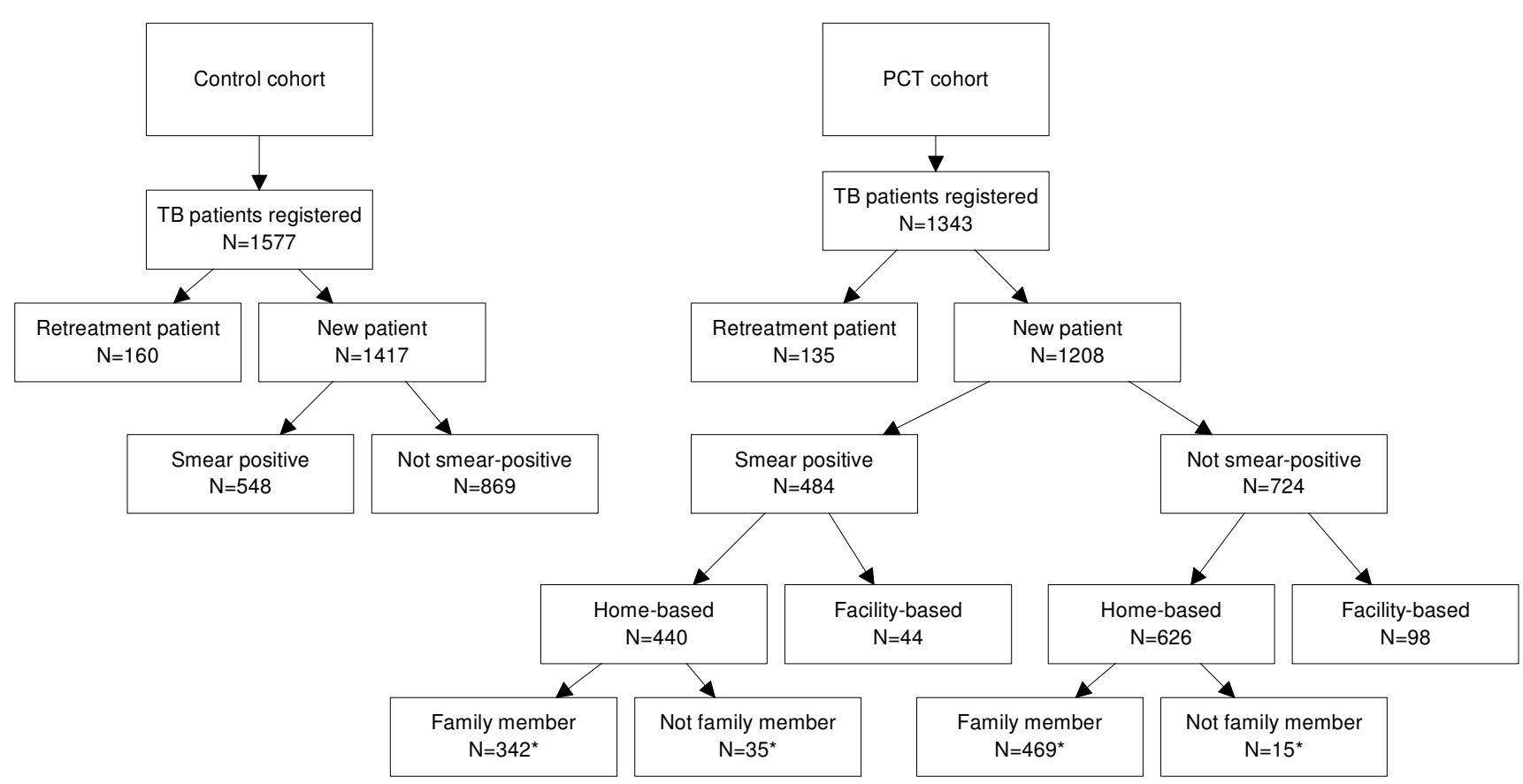

Figure I

Patient disposition. $*$ No information of relationship of treatment supporter for 205 patients.

and the proportion of patients defaulting during treatment. There was no statistically significant difference in the primary outcome (cure) comparing the PCT $(64.5 \%)$ cohort with the control cohort (60.9\%; RR: 1.06 ; $95 \%$ confidence interval $[\mathrm{CI}]: 0.96-1.16 ; P=0.136)$.

Smear-positive patients in the PCT cohort were more likely to have successful treatment $(82.2 \%)$ than patients in the control cohort (69.7\%; RR: 1.18; 95\% CI: $1.10-$

Table I: Baseline characteristics of tuberculosis (TB) patients.

\begin{tabular}{|c|c|c|c|}
\hline & $\begin{array}{l}\text { Historic } \\
n=1417 *\end{array}$ & $\begin{array}{l}P C T \\
n=\mid 208 *\end{array}$ & $P$-value \\
\hline District & & & $<0.001$ \\
\hline Arusha & $394(27.8)$ & $495(4 I .0)$ & \\
\hline Kahama & $394(27.8)$ & $329(27.2)$ & \\
\hline Mfindi & $629(44.4)$ & $384(31.8)$ & \\
\hline Female & $667(47.1)$ & $563(41.9)$ & 0.812 \\
\hline \multicolumn{4}{|l|}{ Age group } \\
\hline$<25$ & $292(20.6)$ & $24 \mid(20.0)$ & 0.796 \\
\hline $25-34$ & $47 \mid(33.2)$ & $390(32.3)$ & \\
\hline $35-44$ & $323(22.8)$ & $260(21.5)$ & \\
\hline$>=45$ & $323(22.8)$ & $290(24.0)$ & \\
\hline Missing & $8(0.6)$ & $27(2.2)$ & \\
\hline Type of TB & & & 0.020 \\
\hline New smear-positive & $548(38.7)$ & $484(40.1)$ & \\
\hline New smear-negative & $473(33.4)$ & $345(28.6)$ & \\
\hline New EPTB & $396(27.9)$ & $379(31.4)$ & \\
\hline
\end{tabular}

*All data are $\mathrm{n}(\%)$.

$\mathrm{PCT}$, patient centred treatment; EPTB, extra pulmonary tuberculosis.
1.26; $P<0.001)$. This was because more patients in the PCT cohort, compared to the historical cohort, completed treatment and had a final smear examination performed at the end of treatment (necessary in order to confirm cure).

Significantly more smear-positive patients in the PCT cohort had a recorded smear conversion at 2 months $(80.2 \%)$, than in the control cohort $(74.6 \%$; RR: 1.07 ; 95\% CI: $1.01-1.15 ; P=0.021)$. This difference was driven by the fact that a larger proportion of patients in the control cohort $(20.1 \%)$ died, transferred, defaulted or did not have a sputum smear performed compared to the PCT cohort $(15.5 \%)$. Comparing the proportion of patients with smear conversion only for those patients who were present at month 2, and had had a smear examination, did not reveal statistically significant changes between the PCT cohort $(93.4 \%)$ and the historic cohort $(94.9 \% ; P=$ $0.359)$.

\section{All new patients}

A comparison of the treatment outcomes for all new patients included in the two cohorts showed a similar finding of a higher success rate in the PCT cohort (79\%) compared to the control cohort (71.6\%); RR: $1.10 ; 95 \%$ CI: $1.05-1.15 ; P<0.001)$.

Comparing treatment delivery strategies in the PCT cohort New smear-positive patients who opted for home-based treatment observation were more likely to be cured com- 
Table 2: Treatment outcomes.

\begin{tabular}{|c|c|c|c|c|c|}
\hline & Historic & PCT & $\mathbf{R R} \dagger$ & $95 \% \mathrm{Cl}$ & $P$-value \\
\hline Smear-positive patients & $n=548^{*}$ & $n=484^{*}$ & & & \\
\hline Smear conversion & $409(74.6)$ & $388(80.2)$ & 1.07 & $1.01--1.15$ & 0.037 \\
\hline Cure & 334 (60.9) & $312(64.5)$ & 1.06 & $0.96-1.16$ & 0.247 \\
\hline Success & $382(69.7)$ & $398(82.2)$ & 1.18 & $1.10--1.26$ & $<0.001$ \\
\hline Death & $92(16.8)$ & $59(12.2)$ & 0.73 & $0.54-0.98$ & 0.042 \\
\hline Default & $28(5.1)$ & $13(2.7)$ & 0.53 & $0.28-0.99$ & 0.055 \\
\hline Transfer out & $41(7.5)$ & $14(2.9)$ & 0.39 & $0.22-0.68$ & 0.001 \\
\hline Fail & $2(0.4)$ & 0 & & & \\
\hline Missing outcome & $3(0.6)$ & 0 & & & \\
\hline All patients & $n=1417^{*}$ & $n=1208^{*}$ & & & \\
\hline Success & $1015(71.6)$ & $954(79.0)$ & 1.10 & $1.05-1.15$ & $<0.001$ \\
\hline Death & $249(17.6)$ & $168(13.9)$ & 0.79 & $0.66-0.95$ & 0.012 \\
\hline Default & $61(4.3)$ & $40(3.3)$ & 0.77 & $0.52--1.14$ & 0.222 \\
\hline Transfer out & $80(5.7)$ & $41(3.4)$ & 0.60 & $0.42-0.86$ & 0.007 \\
\hline Fail & $2(0.1)$ & 0 & & & \\
\hline Missing outcome & $10(0.7)$ & $4(0.3)$ & & & \\
\hline
\end{tabular}

*All data are $\mathrm{n}(\%)$.

† Relative risk comparing PCT cohort with historic cohort.

$\mathrm{Cl}$, confidence interval.

Table 3: Factors associated with cure smear-positive patients in patient centred treatment cohort.

\begin{tabular}{|c|c|c|c|c|c|c|}
\hline & \multicolumn{3}{|c|}{ Univariable } & \multicolumn{3}{|c|}{ Multivariable } \\
\hline & OR & $95 \% \mathrm{Cl}$ & $P$ & OR & $95 \% \mathrm{Cl}$ & $P$ \\
\hline \multicolumn{7}{|l|}{ Treatment observation* } \\
\hline HF-DOT & 1 & & & I & & \\
\hline HB-DOT & 2.37 & $1.27--4.43$ & 0.007 & 2.31 & $1.22--4.38$ & 0.010 \\
\hline \multicolumn{7}{|l|}{ District } \\
\hline Kahama & I & & & 1 & & \\
\hline Arusha & 1.38 & $0.87--2.20$ & 0.169 & 1.27 & $0.79--2.04$ & 0.315 \\
\hline Mufindi & 1.18 & $0.74--1.89$ & 0.482 & 1.04 & $0.65--1.70$ & 0.849 \\
\hline \multicolumn{7}{|l|}{ Supporter characteristics $\dagger$} \\
\hline \multicolumn{7}{|l|}{ Sex } \\
\hline Male & 1 & & & & & \\
\hline Female & 1.19 & $0.75-1.89$ & 0.450 & & & \\
\hline \multicolumn{7}{|l|}{ Relationship } \\
\hline Non-family member & I & & & & & \\
\hline Family member & 1.09 & $0.46-2.61$ & 0.843 & & & \\
\hline \multicolumn{7}{|l|}{ Age } \\
\hline$<25$ years & 1 & & & & & \\
\hline $25-34$ years & 1.06 & $0.50--2.24$ & 0.879 & & & \\
\hline $35--44$ years & 0.96 & $0.42--2.15$ & 0.912 & & & \\
\hline$>=45$ years & 0.84 & $0.38-1.85$ & 0.670 & & & \\
\hline \multicolumn{7}{|l|}{ Education } \\
\hline None & 1 & & & & & \\
\hline Primary education & 1.74 & $0.92-3.27$ & 0.087 & & & \\
\hline Above primary education & 1.64 & $0.69--3.89$ & 0.258 & & & \\
\hline
\end{tabular}

$*$ In all new smear-positive patients $(n=484)$

$\dagger$ In HB-DOT group $(n=440)$

$\mathrm{HF}$, health facility; $\mathrm{HB}$, home-based; DOT, directly observed treatment; $\mathrm{Cl}$, confidence interval; OR, odds ratio. 
pared to patients having treatment observation at the health facility (RR: $2.37 ; 95 \% \mathrm{CI}: 1.27-4.43 ; P=0.007$; Table 3). This was seen in all districts, although the effect was larger in Arusha than in Kahama and Mufindi which, however, did not lead to a statistically significant effect modification in any of the statistical models. Adjusting the effect of home-based treatment for these differences in districts did not change the results (RR: 2.31; 95\% CI: 1.22 - 4.38; $P=0.010)$.

Treatment success in the entire PCT cohort was also more likely to be reported by patients who choose home-based treatment (RR: 4.19; 95\% CI: 2.91 - 6.05; $P<0.001$ ). Again, the effect was larger in Arusha than in the other two districts but did not lead to significant effect modification. Adjustment did not alter the effect of home-based treatment (RR: 4.11; 95\% CI: 2.82 - 6.00; $P<0.001$ ).

\section{Effect of supporters on treatment outcomes}

Of the supporters, $76 \%$ were family members (including spouses), 5\% were non-family members - for $19 \%$, no information on the relationship was available. None of the supporter characteristics was associated either with cure of smear-positive patients (Table 3 ) or treatment success for any of the patients (Table 4). The cure and treatment success rates in patients who had a family member as a supporters were as high as patients who had a nonfamily member as a supporter.

\section{Discussion}

This study showed that treatment outcomes in a cohort of new TB patients receiving treatment under the PCT strategy were comparable to treatment outcomes in patients who received treatment with the conventional health facility-based DOT and administration of loose drugs. The higher success rate in the PCT cohort is driven by a larger proportion of patients completing their treatment. This finding is not easy to interpret. It might be just an effect caused by the fact that the treatment was for 2 months less. For the verifiable outcome cure, there is no difference between the historical cohort and the PCT cohort. In addition, the study did not identify any supporter characteristic that influenced the achievement of cure or treatment success in patients who had their treatment observed at home.

These findings are in line with the results from other studies performed in a variety of settings. A cluster-randomized trial in Nepal showed that $89 \%$ of the patients who had their treatment intake observed by a family member had a treatment success. This was comparable with the $85 \%$ success in patients who were supervised by

Table 4: Factors associated with success all patients in patient centred treatment cohort.

\begin{tabular}{|c|c|c|c|c|c|c|}
\hline & \multicolumn{3}{|c|}{ Univariable } & \multicolumn{3}{|c|}{ Multivariable } \\
\hline & OR & $95 \% \mathrm{Cl}$ & $P$ & OR & $95 \% \mathrm{Cl}$ & $P$ \\
\hline \multicolumn{7}{|l|}{ Treatment observation* } \\
\hline HF-DOT & I & & & I & & \\
\hline HB-DOT & 4.19 & $2.91--6.05$ & $<0.001$ & 4.11 & $2.82--6.00$ & $<0.001$ \\
\hline \multicolumn{7}{|l|}{ District } \\
\hline Kahama & I & & & I & & \\
\hline Arusha & 0.52 & $0.37-0.74$ & $<0.001$ & 1.64 & $1.15--2.33$ & 0.006 \\
\hline Mufindi & 0.49 & $0.36--0.69$ & $<0.001$ & 0.81 & $0.57--1.16$ & 0.262 \\
\hline \multicolumn{7}{|l|}{ Supporter characteristics $†$} \\
\hline \multicolumn{7}{|l|}{ Sex } \\
\hline Male & I & & & & & \\
\hline Female & 0.99 & $0.67--1.47$ & 0.974 & & & \\
\hline \multicolumn{7}{|l|}{ Relationship } \\
\hline Non-family member & I & & & & & \\
\hline Family member & 1.05 & $0.46--2.39$ & 0.910 & & & \\
\hline \multicolumn{7}{|l|}{ Age } \\
\hline$<25$ years & I & & & & & \\
\hline 25 -- 34 years & 0.70 & $0.35--1.43$ & 0.332 & & & \\
\hline 35 -- 44 years & 0.92 & $0.43--1.98$ & 0.845 & & & \\
\hline$>=45$ years & 0.50 & $0.25-1.05$ & 0.067 & & & \\
\hline \multicolumn{7}{|l|}{ Education } \\
\hline None & I & & & & & \\
\hline Primary education & $1.6 \mid$ & $0.92--2.85$ & 0.098 & & & \\
\hline Above primary education & 0.97 & $0.49--1.96$ & 0.950 & & & \\
\hline
\end{tabular}

$*$ In all new patients $(\mathrm{n}=1208)$

$\dagger$ In HB-DOT group $(n=1066)$

$\mathrm{OR}$, odds ratio; $\mathrm{Cl}$, confidence interval; $\mathrm{HF}$, heath facility; $\mathrm{HB}$, home-based. 
a community health worker and higher than the treatment targets from the WHO (85\%) [17]. A retrospective study in Thailand assessed treatment outcomes in patients who accepted direct observation of treatment by staff of the health facility, a village health worker or a family member. Ninety percent of the patients opted for observation by a family member and $10 \%$ for the health staff of the facility. Only one of the 216 patients chose observation by a village health worker. The success rate was higher in patients observed by a family member (87\%) compared to patients observed by health staff (76\%) [15]. A randomized trial in Swaziland reported that treatment observation by family members obtained similar success rates in TB patients as did treatment observation by community health workers (66\% and 68\%, respectively) [18]. Similarly, a randomized trial in Pakistan did not find any differences in treatment success rates in patients observed by family members (62\%), observation at the health facility $(67 \%)$ or self-administration (62\%) [19].

Despite these findings, the ultimate proof of principle for the efficacy of home-based treatment observation is not given by the short-term cure rates or success rate as assessed in this and other studies. Only when, in settings with home-based DOT, the relapse rates do not increase (and preferentially decrease due to improved adherence) can home-based DOT be regarded as effective.

The concept of treatment observation by family members is still controversial within the TB community. The WHO treatment guidelines state that family members should, in general, not be considered as treatment supporters [3]. However, in the updated guidelines on community involvement in TB care, there is a shift towards the idea that there is a need to provide 'an increased range of treatment support options', although this statement was mainly geared at TB patients co-infected with HIV [20].

Despite the above mentioned results of the PCT approach, the success rate in Tanzania remains below the 85\% target set by WHO. This relatively low success rate is mainly driven by the death rate. It is more than likely that this rate is largely a result of co-infection with HIV. It is known that HIV-associated death in TB patients is generally seen at the start of TB-treatment. Even the PCT approach will not have a major impact on these events, which is reflected in a death rate of $12 \%$.

There are several arguments formulated by those who are against the use of family members as treatment supporters. First, family members are not always able to be firm on treatment intake, especially if the patient suffers side effects or if the social context makes it difficult or inappropriate to do so [21]. Second, the design of most studies showing similar, or better, treatment outcomes with observation by family members than by health workers actually test multifaceted interventions including increased monitoring, supervision and training. In the Nepal study, there were frequent visits to the households by research staff in order to monitor the implementation and to collect data. In Thailand, clinic staff were strongly involved in the identification of a treatment supporter and special boxes were prepared in order to facilitate treatment delivery at home [15]. In Senegal, the intervention was clearly multifaceted, including improved communication between health staff and patients and increased training and supervision, making it impossible to tease out the effect of decentralized treatment observation [22]. Third, treatment observation outside the health facility is, in practise, more frequently self administration of treatment. This was shown in a study by a study in Thailand where $11 \%$ of the health personnel did not perform any treatment observation, compared to $23 \%$ of community members and $35 \%$ of family members [23].

The major limitation of the study was the change in design which meant that the present study was not able to adequately test the independent effect of the type of treatment delivery. Instead, it tested a multifaceted intervention. Each part of this intervention might have contributed to the positive findings. In the PCT cohort, all drugs were taken as FDC during the full treatment period. In the control cohort, FDC was only available for isoniazid and rifampicin during the intensive phase. All other drugs were administered as loose drugs. Drug delivery by FDCs might be associated with an improved adherence to therapy [24]. At the same time, the treatment regimen was changed to include rifampicin throughout the duration. An additional 4 months of this potent drug might positively influence the cure rate in the PCT cohort. However, the additional efficacy of this treatment regimen over the conventional regimen used in the control cohort is based largely on the relapse rates and not on the initial cure rates. If the new regimen had an effect on the outcome it was most likely due to its shorter duration and, therefore, the possible higher completion rates, rather than an increased drug efficacy. However, the total intervention will be rolled-out in the rest of the country making the individual contributions of the multifaceted intervention towards the overall positive effect less important. The important finding, that the type of treatment supporter does not influence treatment outcome, makes this nationwide scale-up of the intervention more feasible.

Another limitation was the absence of demographic information for a considerable number of treatment supporters. As mentioned, this did not introduce a bias because of the concurrent start of the study in the districts and the retaining of the freedom of choice for the type of treatment delivery strategy. There is also a marked absence of 
HIV data form the enrolled patients. At the time of the study (2005 for the historical cohort and 2006 of the PCT cohort), routine HIV testing for newly registered patients had not been fully implemented. Collection of this information for inclusion in statistical models would lead to a bias, given the large proportion of patients who would not have this information. A comparison of this information between the two cohorts would also be not possible due to the increase in routine testing between 2005 and 2006.

There is some indication that ascertainment bias might have influenced the results in Arusha. In this district case notification increased considerably during the study and there was, also, a significantly higher probability of cure in the PCT cohort. This may indicate improved diagnosis and/or microscopy. It could also be a result of the quality control measures that were imposed in the PCT cohort but not the historical cohort. However, adjusting the multivariable models did not give any change in the point estimates or the confidence intervals, making this potential bias of little value.

Acceptable and feasible treatment observation strategies are highly context specific [25]. In Tanzania, home-based treatment observation by a supporter of choice is widely appreciated and does not lead to an increase in unfavourable treatment outcomes compared to conventional treatment delivery strategies. It allows patients to rest and recover, as daily health facility DOT places considerable physical strain on patients. Being at home also made it possible for the patient to engage in productive activities and reduce the cost of travel associated with the daily health facility visits. At the same time, it could ease the burden on health facilities [2]. Studies from Malawi and Australia have shown that, in a decentralized setting, adherence to treatment can be as high as seen in healthfacility based treatment observation $[16,26]$. However, it is important to assess adherence to treatment in the setting of Tanzania.

The programmatic condition in which this study was carried out lends strong support to the scaling up of the PCT strategy in Tanzania. This must coincide with rigorous supervision of the health facilities implementing the strategy. Furthermore, follow-up of this first cohort of patients treated under the PCT strategy will give much needed data on the risk of relapse and the resistance patterns in patient who need retreatment: two factors that determine the long-term efficacy of the intervention.

The positive effect of home-based treatment in a programmatic setting found in this study can be of value for areas outside the context of TB. HIV-programmes in especially resource-poor areas are experimenting with such an approach $[27,28]$. The findings that home-based treat- ment can be achieved under programmatic conditions might give the much needed impetus to move from smaller projects to a more general implementation within national treatment programmes.

\section{Conclusions}

The PCT approach showed similar cure rates and better treatment success rates compared to daily health-facility DOT. The results indicate that there are no specific prerequisites for the supporter chosen by the patient. The programmatic setting of the study lends strong support for scaling-up of TB treatment observation outside the health facility.

\section{Abbreviations}

CI: confidence interval; DOT: directly observed therapy; DOTS: DOT, short course; FDC: fixed dose combination; NTLP: National Tuberculosis and Leprosy Programme; PCT: patient centred treatment; RR: risk ratio; TB: tuberculosis; WHO: World Health Organization.

\section{Competing interests}

VH was a full time employee of Novartis Foundation for Sustainable Development during the entire study. At the time of the study design, PG was also a full time employee of the Novartis Foundation for Sustainable Development. None of the other authors declared a competing interest.

\section{Authors' contributions}

SE, NR and FL participated in the design and implementation of the study and provided critical contributions to the drafts of the manuscript. $\mathrm{AM}$ and $\mathrm{AB}$ collected and analysed the data and provided critical contributions to the drafts of the manuscript. HM supervised the data collection and analysis. $\mathrm{VH}, \mathrm{PG}$ and FC participated in the design of the study and provided critical contributions to the drafts of the manuscript. FvL participated in the design of the study, assisted in data analysis and wrote the first and all consecutive drafts of the manuscript.

\section{Acknowledgements}

The study was funded by the Novartis Foundation for Sustainable Development, Switzerland. The funder of the study was involved in study design and data interpretation. Data collection and data analysis were performed independently of the funder. The corresponding author had access to all data in the study. The final decision to submit for publication was made by the NTLP of Tanzania. FvL received funding towards this work from the UK Department for International Development (DFID) for the benefit of developing countries. The views expressed are not necessarily those of DFID.

\section{References}

I. World Health Organization: Global Tuberculosis Control: Surveillance, Planning, Financing. Geneva: WHO; 2008.

2. Cobelens FG, Egwaga SM, van Ginkel T, Muwinge H, Matee MI, Borgdorff MW: Tuberculin skin testing in patients with HIV infection: limited benefit of reduced cutoff values. Clin Infect Dis 2006, 43(5):634-639. 
3. World Health Organization: Treatment of Tuberculosis: Guidelines for National Programmes 3rd edition. Geneva: WHO; 2003.

4. Enarson DA, Rieder HL, Arnadottir T, Trébucq A: Management of Tuberculosis: A Guide for Low Income Countries. 5th edition. Paris: International Union Against Tuberculosis and Lung Disease; 2000.

5. World Health Organization: Anti-tuberculosis Drug Resistance in the World: Fourth Global Report. Geneva: WHO; 2008.

6. Blondal K: Barriers to reaching the targets for tuberculosis control: multidrug-resistant tuberculosis. Bull World Health Organ 2007, 85(5):387-390. discussion 391-384

7. Churchyard G], Corbett EL, Kleinschmidt I, Mulder D, De Cock KM Drug-resistant tuberculosis in South African gold miners: incidence and associated factors. Int J Tuberc Lung Dis 2000 , 4(5):433-440.

8. Burman W, Benator D, Vernon A, Khan A, Jones B, Silva C, Lahart C Weis $S$, King B, Mangura B, et al.: Acquired rifamycin resistance with twice-weekly treatment of HIV-related tuberculosis. Am J Respir Crit Care Med 2006, I 73(3):350-356.

9. El-Sadr WM, Perlman DC, Denning E, Matts JP, Cohn DL: A review of efficacy studies of 6-month short-course therapy for tuberculosis among patients infected with human immunodeficiency virus: differences in study outcomes. Clin Infect Dis 200I, 32(4):623-632.

10. Whalen CC, Johnson JL, Okwera A, Hom DL, Huebner R, Mugyenyi $P$, Mugerwa RD, Ellner J]: A trial of three regimens to prevent tuberculosis in Ugandan adults infected with the human immunodeficiency virus. Uganda-Case Western Reserve University Research Collaboration. N Engl J Med 1997, 337( ( 2):80I-808.

11. Korenromp EL, Scano F, Williams BG, Dye C, Nunn P: Effects of human immunodeficiency virus infection on recurrence of tuberculosis after rifampin-based treatment: an analytical review. Clin Infect Dis 2003, 37(I): I0I-1 I2.

12. Wandwalo E, Kapalata N, Egwaga S, Morkve O: Effectiveness of community-based directly observed treatment for tuberculosis in an urban setting in Tanzania: a randomised controlled trial. Int J Tuberc Lung Dis 2004, 8( I 0): I 248-I254.

13. Lwilla F, Schellenberg D, Masanja H, Acosta C, Galindo C, Aponte J, Egwaga S, Njako B, Ascaso C, Tanner M, et al.: Evaluation of efficacy of community-based vs. institutional-based direct observed short-course treatment for the control of tuberculosis in Kilombero district, Tanzania. Trop Med Int Health 2003, 8(3):204-210.

14. Adatu F, Odeke R, Mugenyi M, Gargioni G, McCray E, Schneider E Maher D: Implementation of the DOTS strategy for tuberculosis control in rural Kiboga District, Uganda, offering patients the option of treatment supervision in the community, 1998-1 999. Int J Tuberc Lung Dis 2003, 7(9 SuppI I):S63-7I.

15. Akkslip S, Rasmithat S, Maher D, Sawert H: Direct observation of tuberculosis treatment by supervised family members in Yasothorn Province, Thailand. Int J Tuberc Lung Dis 1999, 3(12): 1061-1065

16. Manders AJ, Banerjee A, Borne HW van den, Harries AD, Kok GJ, Salaniponi FM: Can guardians supervise TB treatment as wel as health workers? A study on adherence during the intensive phase. Int J Tuberc Lung Dis 200I, 5(9):838-842.

17. Newell JN, Baral SC, Pande SB, Bam DS, Malla P: Family-member DOTS and community DOTS for tuberculosis control in Nepal: cluster-randomised controlled trial. Lancet 2006, 367(95 I 4): $903-909$.

18. Wright J, Walley J, Philip A, Pushpananthan S, Dlamini E, Newell J Dlamini S: Direct observation of treatment for tuberculosis: a randomized controlled trial of community health workers versus family members. Trop Med Int Health 2004, 9(5):559-565.

19. Walley JD, Khan MA, Newell JN, Khan MH: Effectiveness of the direct observation component of DOTS for tuberculosis: a randomised controlled trial in Pakistan. Lancet 2001, 357(9257):664-669

20. World Health Organization: Community involvement in tuberculosis care and prevention. Geneva: WHO; 2008.

21. Frieden TR, Sbarbaro JA: Promoting adherence to treatment for tuberculosis: the importance of direct observation. Bull World Health Organ 2007, 85(5):407-409.

22. Thiam S, LeFevre AM, Hane F, Ndiaye A, Ba F, Fielding KL, Ndir M, Lienhardt $C$ : Effectiveness of a strategy to improve adherence to tuberculosis treatment in a resource-poor setting: a cluster randomized controlled trial. JAMA 2007, 297(4):380-386.

23. Pungrassami P, Johnsen SP, Chongsuvivatwong V, Olsen J, Sorensen HT: Practice of directly observed treatment (DOT) for tuberculosis in southern Thailand: comparison between different types of DOT observers. Int J Tuberc Lung Dis 2002, 6(5):389-395

24. Blomberg B, Spinaci S, Fourie B, Laing R: The rationale for recommending fixed-dose combination tablets for treatment of tuberculosis. Bull World Health Organ 200I, 79(I):6I-68.

25. Macq JC, Theobald S, Dick J, Dembele M: An exploration of the concept of directly observed treatment (DOT) for tuberculosis patients: from a uniform to a customised approach. Int J Tuberc Lung Dis 2003, 7(2): 103-109.

26. Maclntyre CR, Goebel K, Brown GV, Skull S, Starr M, Fullinfaw RO A randomised controlled clinical trial of the efficacy of family-based direct observation of anti-tuberculosis treatment in an urban, developed-country setting. Int J Tuberc Lung Dis 2003, 7(9):848-854.

27. Abaasa AM, Todd J, Ekoru K, Kalyango JN, Levin J, Odeke E, Karamagi CA: Good adherence to HAART and improved survival in a community HIVIAIDS treatment and care programme: the experience of The AIDS Support Organization (TASO), Kampala, Uganda. BMC Health Serv Res 2008, 8:24I.

28. Weidle PJ, Wamai N, Solberg P, Liechty C, Sendagala S, Were W, Mermin J, Buchacz K, Behumbiize P, Ransom RL, et al.: Adherence to antiretroviral therapy in a home-based AIDS care programme in rural Uganda. Lancet 2006, 368: I587-I594.

\section{Pre-publication history}

The pre-publication history for this paper can be accessed here:

http://www.biomedcentral.com/1741-7015/7/80/prepub
Publish with Biomed Central and every scientist can read your work free of charge

"BioMed Central will be the most significant development for disseminating the results of biomedical research in our lifetime. "

Sir Paul Nurse, Cancer Research UK

Your research papers will be:

- available free of charge to the entire biomedical community

- peer reviewed and published immediately upon acceptance

- cited in PubMed and archived on PubMed Central

- yours - you keep the copyright

Submit your manuscript here:

http://www.biomedcentral.com/info/publishing_adv.asp
BioMedcentral 